\title{
Foreign Body Removal by Snare Loop: During Intracranial Stent Procedure
}

\author{
Yul Oh, MD', Dae Hyun Hwang, MD', Young Hwan Ko, MD', Ik Won Kang, MD', \\ In Soo Kim, MD², Choon Woong Hur, MD²
}

We present a case of successful retrieval of an intracranial stent using a snare wire. A 52-year-old woman presented with left border zone infarction. On cerebral angiography, the C6 segment of the left internal carotid artery (ICA) showed significant stenosis. We attempted stenting of the lesion, although stent dislodgement occurred in the ICA C4 segment. We successfully removed it using a snare loop, and there were no complications during the procedure.

Key Words : Foreign body; Snare loop; Endovascular procedures

Stent detachment and loss from the balloon is one of the complications of intracranial interventional procedures. Embolization of the detached stent is associated with a high risk of intracranial vessel occlusion and subsequent cerebral infarction. Therefore, interventional radiologists may be confronted with the problem of how best to retrieve intravascular foreign bodies, including detached stents [1].

We present a case of successful retrieval of an intracranial stent using a snare wire. To our knowledge, this is the first case report of intracranial stent removal.

\footnotetext{
'Department of Radiology, Hallym University Hangang Sacred Heart Hospital, Seoul, Korea

2Department of Neurosurgery, Myungji St. Mary's Hospital, Seoul, Korea

Received September 24, 2011; accepted

after revision November 7, 2011.

Correspondence to: Dae Hyun Hwang, MD, Department of Radiology, Hangang Sacred Heart Hospital, 94-200 Yeongdeungpodong 2-ga, Yeongdeungpo-gu, Seoul 150-719, Korea.

Tel. 82.2.2639.5225 Fax. 82.2.2679.0121

E-mail: mddhhwang@naver.com

This is an Open Access article distributed under the terms of the Creative Commons Attribution Non-Commercial License (http://creativecommons.org/licenses/by-nc/3.0) which permits unrestricted non-commercial use, distribution, and reproduction in any medium, provided the original work is properly cited.
}

\section{CASE REPORT}

A 52-year-old woman was admitted to our unit because of a headache. Contrast-enhanced computed tomography $(\mathrm{CT})$ revealed a cerebral infarction in the left border zone. Diagnostic angiography of the internal carotid artery (ICA) was performed using a femoral approach and showed significant stenosis in the ICA C6 segment (Fig. 1A). For management of this lesion, we chose endovascular recanalization therapy.

A $6 \mathrm{~F}$ guiding catheter was inserted into the ICA where we placed 0.012 inch wire. However, this wire passed into an extraluminal dissection plane. A 0.014 inch wire was then introduced in order to be placed in the ICA and it was easily passed through the original stenotic site. This wire was then left in place and a 0.035 inch, stiff wire was passed in parallel fashion. This stiff wire appeared to take a difficult course toward the outer curvature of the vessel and crossed over the first wire. The target lesion was then predilated using a $2.5 \times 20 \mathrm{~mm}$ Ryujin balloon (Terumo, Torkyo, Japan) inflated at 10 atms (Fig. 1B). A $4.0 \times 20-\mathrm{mm}$ Neuroform stent (Boston Scientific/Target, Fremont, CA, U.S.A.) was delivered to the ICA. The implanted stent was post-dilated with a very good angiographic result. However, when removing the stiff guide wire, 


\section{Foreign Body Removal by Snare Loop}

stent dislodgement occurred, and the stent became localized in the ICA C4 segment (Fig. 1C). Stent evacuation was then performed using a snare loop (Fig. 1D), and the stent was successfully retrieved (Fig. 1E). After foreign body removal, angiogram shows no significant complications in the distal ICA (Fig. 1F).

\section{DISCUSSION}

With the increasing use of indwelling catheters and interventional devices, the likelihood of stent loss or dislodgement may increase as a complication of an interventional procedure. Stent loss is most likely to occur during withdrawal of a balloon catheter with a
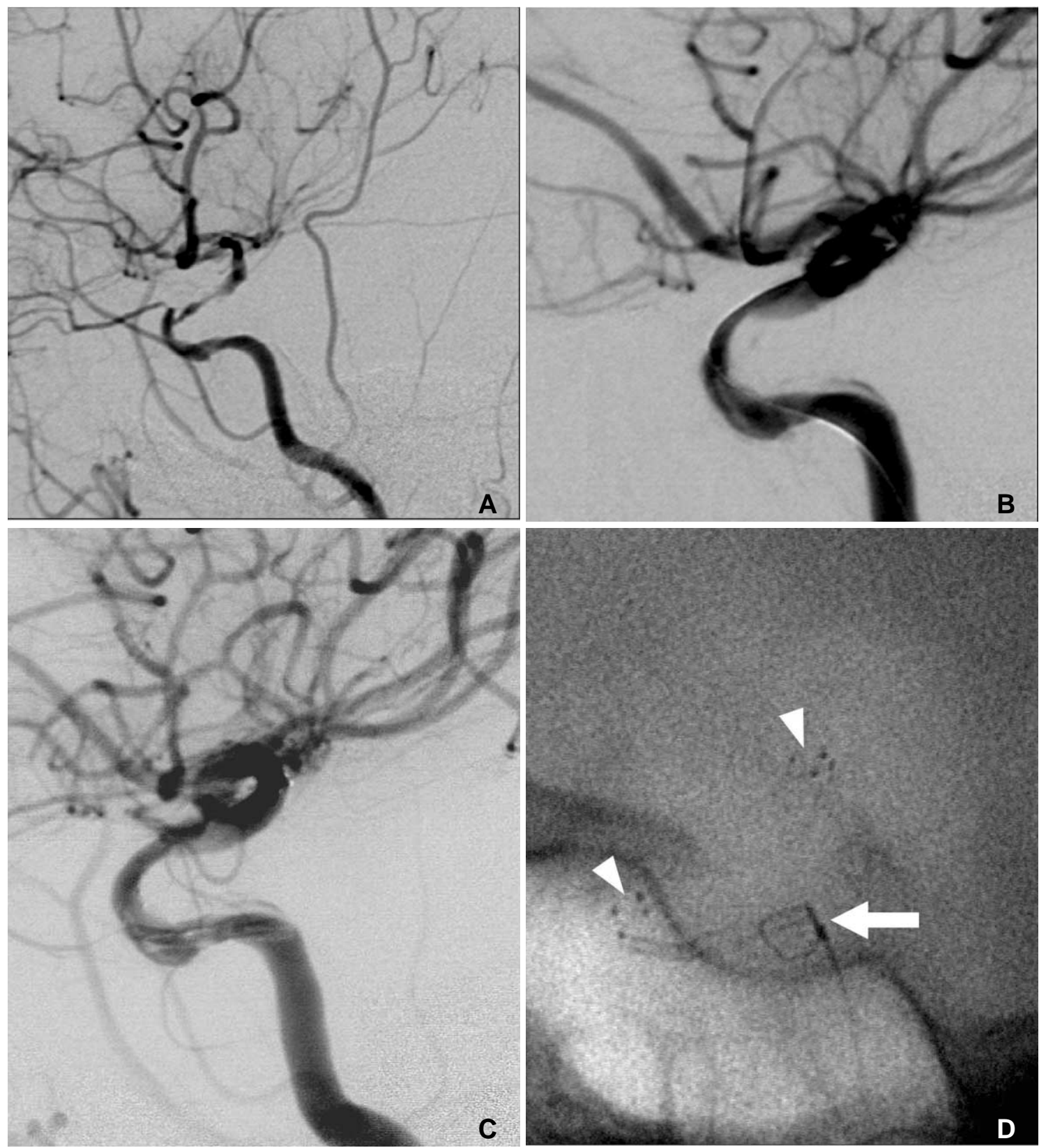

Fig. 1. A. Cerebral angiography shows severe stenosis of the left internal carotid artery (ICA) C6 segment.

B. Control angiography shows partial recanalization of stenotic segment afeter balloon angioplasty using a $2.5 / 20 \mathrm{~mm}$ Ryujin balloon (Terumo, Japan).

C. A $4.0 \times 20 \mathrm{~mm}$ Neuroform stent was delivered to the distal ICA. However, a dislodgement of the stent occurred and the stent was localized in the ICA C4 segment.

D. Stent evacuation was performed using a snare loop. On fluoroscopy, arrowheads indicate proximal and distal marker of Neuroform stent, and the arrow indicates a snare loop.

Continued 

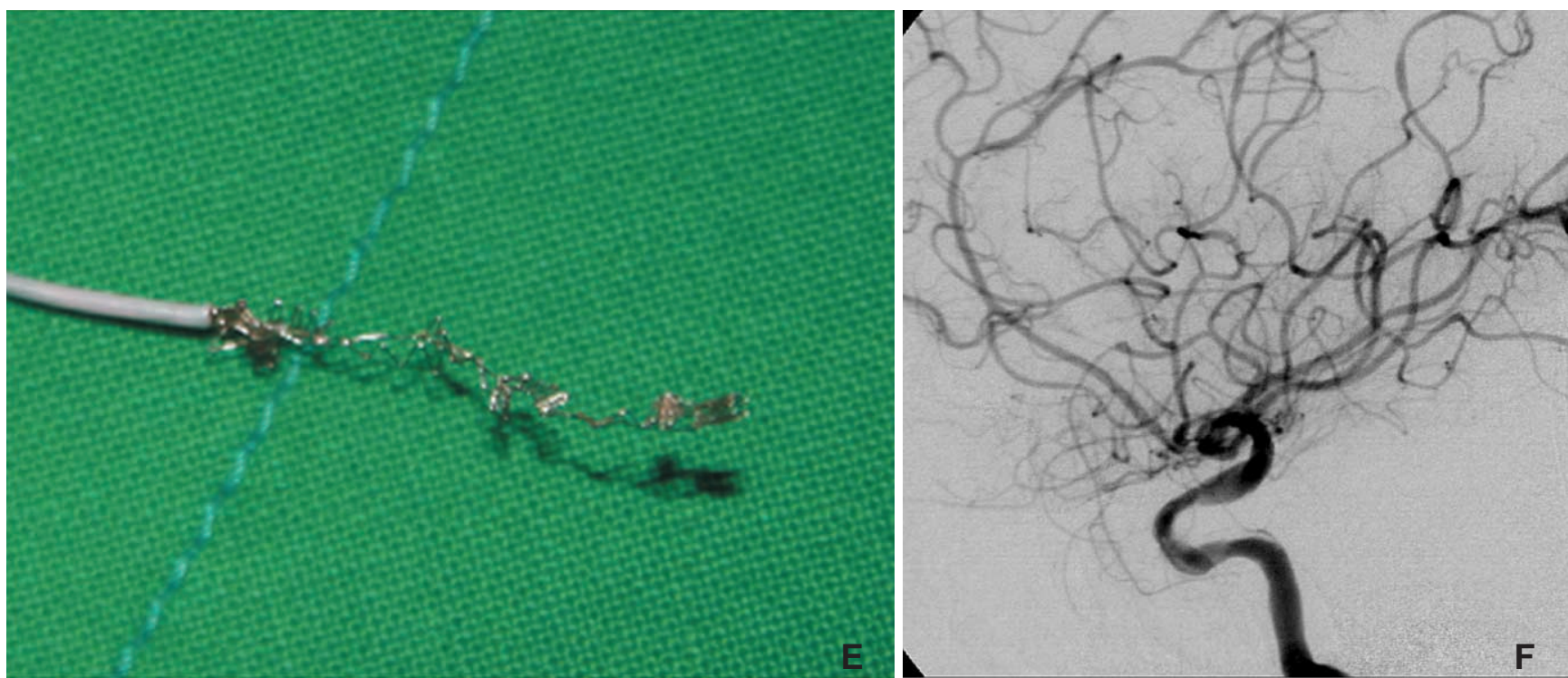

Fig. 1. E. The stent was successfully retrieved.

F. Follow-up left ICA angiogram shows no significant complications in the distal ICA.

stent to the guiding catheter if the lesion could not be reached or if the stenosis could not be passed with the stent and balloon catheter [2, 3]. Furthermore, inadequate control of a therapeutic catheter with a guiding catheter or the guiding catheter falling out as a result of its shallow introduction to the artery, increase the risk rate of slip-off and stent loss. It has also been reported that direct stenting without predilatation or introduction of a stent through another stent, are also risk factors for incorrect stenting and misplacement [4].

Leaving the dislocated stent in situ may result in hazardous complications such as traumatic injury to the vascular wall with the subsequent threat of perforation, local thrombogenicity and ischemia [5]. In order to prevent procedure-related complications from occurring, retrieval of misplaced stents should be attempted. The loop-snare, balloon-catheter technique, two-wire technique, basket and forceps have been used to retrieve intravascular foreign bodies including misplaced stents [6].

As a case of intracranial stent dislodgement has never been reported, it was, therefore, difficult to determine which interventional procedure we should attempt in order to remove the stent and how serious the complications might be. We decided to retrieve the stent using the snare-loop system because the snare loop is the first choice for retrieving foreign bodies from vessel [5-8], and it is relatively safe and easy to use and it has low complication rates. However, the choice of the retrieval technique should be specific for each case and based on the operator's experience. In our case, as the misplaced stent had not been deployed and did not ride on a guidewire, a snare loop seemed to be the best method of retrieval.

Interventional radiologists should keep in mind how to manage this complication when doing procedures. However, prevention of such procedure-associated complications is more important and effective in order to assure the least patient morbidity and mortality. The way to avoid this complication is very simple. The size of the guiding catheter, microwire, balloon, and stent should be checked before the procedure is started. Also, when considering which size devices should be used, the diameters of the stent and guidewire should be rechecked.

\section{References}

1. Patel T, Shah S, Pandya R, Sanghvi K, Fonseca K. Broken guidewire fragment: a simplified retrieval technique. Catheter Cardiovasc Interv 2000;51:483-486

2. Eggebrecht H, Haude M, von Birgelen C, Oldenburg O, Baumgart D, Herrmann J, et al. Nonsurgical retrieval of embolized coronary stents. Catheter Cardiovasc Interv 2000;51:432-440

3. Chu CS, Lee ST, Lee KT, Lin TH, Lin CT, Voon WC, et al. Successful retrieval of dislodged paclitaxil-eluting stent with a nitinol loop snare: a case report. Kaohsiung J Med Sci 2005;21: 566-570

4. Juszkat R, Dziarmaga M, Zabicki B, Bychowiec B. Successful coronary stent retrieval from the renal artery: a case report. Cardiol J 2007; 12:87-90

5. Koseoglu K, Parildar M, Oran I, Memis A. Retrieval of intravascular foreign bodies with goose neck snare. Eur J Radiol 2004; 


\section{Foreign Body Removal by Snare Loop}

49:281-285

6. Gabelmann A, Kramer S, Gorich J. Percutaneous retrieval of lost or misplaced intravascular objects. AJR Am J Roentgenol 2001; 176:1509-1513

7. Cekirge S, Weiss JP, Foster RG, Neiman HL, McLean GK.
Percutaneous retrieval of foreign bodies: experience with the nitinol Goose Neck snare. J Vasc Interv Radiol 1993;4:565-568

8. Juszkat R, Dziarmaga M, Zabicki B, Bychowiec B. Successful coronary stent retrieval from the renal artery. Cardiol $J$ 2007; $14: 87-90$ 